\title{
Microscopic Findings Specimen Type
}

National Cancer Institute

\section{Source}

National Cancer Institute. Microscopic Findings Specimen Type. NCI Thesaurus. Code C117591.

The type of a material sample taken from a biological entity for microscopic testing. 